\title{
Dopamine metabolism by a monoamine oxidase mitochondrial shuttle activates the electron transport chain
}

\author{
Steven M. Graves ${ }^{1}$, Zhong Xie ${ }^{1}$, Kristen A. Stout ${ }^{1}$, Enrico Zampese ${ }^{1}$, Lena F. Burbulla ${ }^{2}$, \\ Jean C. Shih ${ }^{5}$, Jyothisri Kondapalli ${ }^{1}$, Tomasso Patriarchi ${ }^{6}$, Lin Tian ${ }^{6}$, Lars Brichta ${ }^{3}$, Paul \\ Greengard $^{3}$, Dimitri Krainc ${ }^{2}$, Paul T. Schumacker ${ }^{4}$, D. James Surmeier ${ }^{1}$ \\ ${ }^{1}$ Department of Physiology, Feinberg School of Medicine, Northwestern University, Chicago, IL \\ USA \\ ${ }^{2}$ Department of Neurology, Feinberg School of Medicine, Northwestern University, Chicago, IL \\ USA \\ ${ }^{3}$ Laboratory of Molecular and Cellular Neuroscience, Rockefeller University, New York, New York, \\ USA \\ ${ }^{4}$ Department of Pediatrics, Feinberg School of Medicine, Northwestern University, Chicago, IL \\ USA \\ ${ }^{5}$ Department of Pharmacology and Pharmaceutical Sciences, School of Pharmacy, University of \\ Southern California, Los Angeles, CA 90089-9121 \\ ${ }^{6}$ Department of Biochemistry and Molecular Medicine, School of Medicine, University of \\ California, Davis, CA USA
}

\section{SUMMARY}

Monoamine oxidase (MAO) metabolizes cytosolic dopamine (DA) thereby limiting autooxidation, but is also thought to generate cytosolic hydrogen peroxide $\left(\mathrm{H}_{2} \mathrm{O}_{2}\right)$. We show that MAO metabolism of DA does not increase cytosolic $\mathrm{H}_{2} \mathrm{O}_{2}$, but leads to mitochondrial electron transport chain (ETC) activity. This was dependent upon MAO anchoring to the outer mitochondrial membrance and shuttling of electrons through the intermembrane space to support the bioenergetic demands of phasic DA release.

Monoamine oxidases (MAOs) are oxidoreductases that deaminate catecholamines. In dopaminergic axons, released dopamine (DA) is taken up from the extracellular space, where it is recycled into vesicles or degraded by MAO. The biological rationale for cytosolic

Users may view, print, copy, and download text and data-mine the content in such documents, for the purposes of academic research, subject always to the full Conditions of use:http://www.nature.com/authors/editorial_policies/license.html\#terms

Correspondence: D. James Surmeier, Ph.D., Department of Physiology, Feinberg School of Medicine, Northwestern University, 303 E. Chicago Ave., Chicago, IL 60611 USA, j-surmeier@northwestern.edu, 312-503-4904.

Author contributions: Conceptualization: DJS, SMG, KAS, PTS, ZX, DK; Methodology: DJS, SMG, PTS, KAS, ZX; Investigation:

SMG, KAS, ZX, EZ, LFB, LB; Formal Analysis: SMG, KAS, ZX, LB; Writing original draft: SMG and DJS; Reviewing and Editing: SMG, ZX, EZ, LFB, JCS, JK, KAS, LB, PG, DK, PTS, DJS; Funding acquisition: SMG, DK, PG and DJS; Resources: JK, LB, PG,

JCS, LT, TP; Supervision: DJS.

Conflict of interest: The authors declare no competing interests.

Accession codes: There are no accession codes associated with data presented. 
DA metabolism is unknown. One hypothesis is that MAO limits the generation of damaging quinones by DA auto-oxidation in the cytosol ${ }^{1}$. However, it is also currently thought that the electrons generated by MAO activity are transferred to molecular oxygen, resulting in the generation of cytosolic hydrogen peroxide $\left(\mathrm{H}_{2} \mathrm{O}_{2}\right)$ that could damage proteins and lipids, particularly in axons where DA release and recycling are prominent ${ }^{2,3}$.

To investigate this puzzling scenario, genetically encoded, $\mathrm{H}_{2} \mathrm{O}_{2}$-sensitive, optical thiol redox sensors ${ }^{4}$ were expressed in subcellular compartments of dopaminergic neurons and pharmacological tools used to acutely drive DA metabolism by MAO. First, a viral vector carrying an expression construct for a cytosolic redox-sensitive variant of green fluorescent protein (cyto-roGFP) was injected into the substantia nigra pars compacta $(\mathrm{SNc})$ of mice. After infection, cyto-roGFP expression was evident throughout dopaminergic cell bodies, dendrites and axons (Extended Data Figure 1A). Ex vivo brain slices were prepared and twophoton laser scanning microscopy (2PLSM) used to measure the thiol redox status of SNc DA neurons, which is altered by $\mathrm{H}_{2} \mathrm{O}_{2}$ and/or quinone generation. Unexpectedly, acute bath application of methamphetamine (10 $\mu \mathrm{M}$; applied for 10-20 min), which increases cytosolic DA by disrupting vesicular monoamine transporters (VMATs) ${ }^{5,6}$, failed to increase cytosolic oxidation in axons (the primary DA release site) or cell bodies (Extended Data Figures 1F, G). The DA precursor levodopa ( $100 \mu \mathrm{M}$; applied for $>30 \mathrm{~min})$, which elevates cytosolic DA by increasing synthesis ${ }^{7}$, also had no measurable effect on cytosolic oxidation in this time frame (Extended Data Figures 1F, H).

Although acutely increasing cytosolic DA did not affect cytosolic redox status, it increased axonal mitochondrial matrix thiol oxidation measured with a variant of roGFP that was targeted to the mitochondrial matrix (mito-roGFP) (Figure 1A; Extended Data Figure 1B). This effect on mitochondria was robust in axons (Figure 1B-E), a region where DA is released and content is known to be high, but was absent in the cell body (Extended Data Figure 2A). Reserpine treatment of mice ( $5 \mathrm{mg} / \mathrm{kg}$ once daily for 5 days), which depletes vesicular DA, prevented the mitochondrial redox effects of methamphetamine (Figure 1F). Compounds that block the plasma membrane DA transporter, but do not inhibit VMATs (i.e. cocaine and methylphenidate), had no effect on mitochondrial matrix redox status (Extended Data Figure 2B).

Why might elevations in axonal DA increase mitochondrial, but not cytosolic, oxidant stress? MAO is anchored to the outer membrane of mitochondria, so it is possible that electrons arising from metabolism of DA are not directly transferred to oxygen, but are shuttled through the intermembrane space to the electron transport chain (ETC). In this way, DA metabolism by MAO could contribute to the inner membrane potential, which is used to produce adenosine triphosphate (ATP).

As a first step toward testing this hypothesis, the type of MAO expressed by SNc dopaminergic neurons was determined. In agreement with work in human dopaminergic neurons $^{8}$, bacTRAP profiling of messenger ribonucleic acid (mRNA) in mouse SNc dopaminergic neurons detected both MAO-A and MAO-B messenger ribonucleic acids (mRNAs), with MAO-A mRNA being the more abundant (MAO-A: 19.76 \pm 0.54 vs. MAOB: $4.49 \pm 0.48$ mean in reads per kilobase per million; $n=6$ mice). When brain slices were pre- 
incubated with the MAO-B inhibitor rasagiline, the effects of elevating cytosolic DA on mitochondrial oxidation were attenuated (Figure 1C, E). The MAO-A inhibitor clorgyline also diminished the mitochondrial redox effects of increasing cytosolic DA (Extended Data Figure 2C). Neither rasagiline nor clorgyline altered basal mitochondrial matrix redox status, presumably reflecting low basal MAO activity in axons of ex vivo brain slices (Extended Data Figure 2D). However, acutely elevating cytosolic DA in the presence of rasagiline caused cytosolic thiol oxidation status to increase (Extended Data Figures 1G, H), suggesting that when MAO is inhibited, DA auto-oxidized.

To ensure that the actions of rasagiline and clorgyline were not a result of 'off-target' effects, these experiments were repeated in mice in which both MAO-A and MAO-B had been deleted $^{9}$. Elevating cytosolic DA had no effect on axonal mitochondrial redox status in DA neurons within brain slices from these double knockout mice (Figure 1G, H). An attempt to rescue MAO activity by expression of a mutant form of MAO-B lacking the mitochondrial tethering sequence in knockout dopaminergic neurons yielded an increase in cytosolic (Extended Data Figure 1I), but not mitochondrial (Extended Data Figure 2E), oxidant stress in response to a release of DA from vesicular stores.

To determine if DA metabolism by $\mathrm{MAO}$ at the outer mitochondrial membrane generates $\mathrm{H}_{2} \mathrm{O}_{2}$ that affects redox status in the nearby matrix but not the cytosol, a variant of roGFP was generated with the mitochondrial tethering peptide sequence from the $\mathrm{C}$-terminus of MAO. This placed the redox sensor in close physical proximity to MAO in dopaminergic neurons (Extended Data Figures 1C-E). Subsequent release of vesicular DA (by acute bath application of $10 \mu \mathrm{M}$ methamphetamine) did not detectably increase thiol oxidant status at the surface of mitochondria unless MAO was inhibited (Figure 1I, J). In this situation, autooxidation of DA leading to the generation of $\mathrm{H}_{2} \mathrm{O}_{2}$ is likely to be responsible for the roGFP signal at the mitochondrial surface.

To verify that the ability of cytosolic DA to increase matrix oxidation was not peculiar to mouse dopaminergic neurons, human dopaminergic neurons were differentiated from induced pluripotent stem cells (iPSCs). Increasing DA synthesis by acute exposure to levodopa increased mitochondrial matrix roGFP oxidation in human dopaminergic neurons in a MAO-dependent manner, demonstrating the generality of the phenomenon (Extended Data Figure 2F).

Where do the electrons generated by mitochondrially tethered MAO metabolism of DA go? Mitochondria are biosynthetic 'engines' that use reducing equivalents to drive proton pumping by the ETC, creating an electrochemical gradient across the inner mitochondrial membrane. This gradient is used by complex V to convert adenosine diphosphate (ADP) to ATP. For the electrons generated by MAO to reach the ETC, they would have to traverse the intermembrane space. This is plausible given that MAO binds flavin adenine dinucleotide (FAD), which can act as an electron shuttle, and many of the proteins that reside within the intermembrane space are capable of shuttling electrons ${ }^{10}$ (Figure 2A). To test the hypothesis that MAO activity increases the flux of electrons through the intermembrane space, a roGFP expression construct with an intermembrane space targeting sequence ${ }^{11}$ was introduced into human dopaminergic neurons. After allowing expression of the protein (Figure 2B), neurons 
were exposed to levodopa for approximately 30 minutes to boost DA synthesis. This acute exposure to levodopa robustly increased oxidation of roGFP within the intermembrane space in an MAO-dependent manner (Figure 2C).

If MAO-derived electrons are shuttled to complex IV, then MAO activity should increase proton pumping and contribute to the maintenance of the inner mitochondrial membrane (IMM) potential. To test this hypothesis, the cationic dye tetramethylrhodamine (TMRM) was used to monitor IMM potential (Figure 2D). Complex III was inhibited to block electron flux from earlier ETC complexes, and the adenine nucleotide translocase (ANT) was inhibited to prevent cytosolic ATP from driving complex V in reverse. As expected, inhibition of complex III and ANT caused TMRM fluorescence to fall, confirming that inhibition of the ETC and complex V dissipates the IMM potential. The trajectory of the fall was fit mathematically (Extended Data Figure 3). In agreement with the proposition that MAO metabolism of DA was contributing to complex IV activity, boosting cytosolic DA levels consistently slowed the decline in TMRM fluorescence (Figure 2E).

What are the functional consequences of MAO-catalyzed electron shuttling to the mitochondria? If electrons are shuttled to the ETC, then MAO activity could increase mitochondrial ATP production. To test this the cytosolic ATP/ADP ratio was monitored using PercevalHR ${ }^{12}$. In human (Figure 3A, B) and mouse (Figure 3C,D) dopaminergic axons, acutely increasing cytosolic DA increased the ATP/ADP ratio in a MAO-dependent manner. Using repetitive electrical stimulation to emulate sustained neuronal activity it was revealed that inhibiting MAO-B activity diminished cytosolic ATP levels, although not as profoundly as the complex V inhibitor oligomycin $(10 \mu \mathrm{M})$ (Figure 3E-H). However, the contribution of MAO-B to mitochondrial ATP production was transient, manifesting itself at the beginning of the stimulus train, but not later (Figure 3G, H). Because the affinity of VMAT2 for DA is several hundred fold greater than that of MAO-B ${ }^{13,14}$, our interpretation of this result is that as cytosolic DA falls with repetitive stimulation, VMAT2 out-competes MAO-B for DA, diminishing the impact of MAO-B metabolism on mitochondrial ATP generation.

Taken together, these observations suggest that when cytosolic DA is abundant, electrons generated by MAO-B deamination of DA help axonal mitochondria meet the bioenergetic needs associated with transmitter release. To test this hypothesis, DA release was monitored using the genetically-encoded optical sensor dLight (Figure 3I ${ }^{15}$. DA release was evoked with either a single electrical pulse or a five pulse burst $(100 \mathrm{~Hz})$. Interestingly, DA release in response to a single stimulus was not affected either by MAO-B inhibition or oligomycin (Figure 3J, K). This lack of mitochondrial dependence is consistent with recent work in glutamatergic neurons showing that glycolysis is capable of providing the energy necessary for a single release event ${ }^{16}$. However, as in other neurons ${ }^{16}$, DA release in response to a short train of stimuli was dependent upon mitochondrial ATP production and MAO-B (Figure 3J, K); similar results were obtained using cyclic voltammetry to measure DA release (Extended Data Figure 4). Thus, axonal MAO-B metabolism contributed to the bioenergetic requirements for sustained DA release. 
MAO is thought to prevent neuronal oxidative stress by limiting DA-quinone formation, yet paradoxically induces stress by releasing $\mathrm{H}_{2} \mathrm{O}_{2}$ in the cytosol. Our findings challenge this view, revealing that electrons generated by MAO deamination of DA are shuttled to the ETC, contributing to inner membrane polarization and helping to sustain DA release by increasing ATP production. This arrangement provides a biological rationale for the tethering of MAO to the outer membrane of mitochondria.

In vivo, the autonomous pacemaking of dopaminergic neurons is interrupted by bursts of spiking that convey environmental information and facilitate motor control ${ }^{17-19}$. During these bursts, DA reuptake could result in cytosolic DA accumulation. In those periods, MAO metabolism of DA could limit auto-oxidation, but also could drive ATP generation to sustain the DA release needed to mobilize basal ganglia circuits and movement ${ }^{20}$.

\section{METHODS}

\section{Animals}

Male mice expressing the redox sensitive roGFP probe targeting the mitochondrial matrix under the tyrosine hydroxylase regulatory element ${ }^{21}$, monoamine oxidase A/B knockout ${ }^{9}$, DAT bacTRAP 22 and wild-type mice (C57/B16) were bred in-house and used with approval by the Northwestern and Rockefeller University Animal Care and Use Committees and in accordance with the National Institutes of Health Guide for the Care and Use of Laboratory Animals. Animals were group housed with food and water provided ad libitum on a 12 hour light/dark cycle and sacrificed between 5 and 10 weeks of age for all experiments with the exception of DAT bacTRAP mice used for sequencing which were sacrificed at 4 months of age.

\section{Stereotaxic surgery}

PCR amplified roGFP, mitoroGFP, truncated MAO-B lacking C-terminus tail sequence required for anchoring (Amino acids 492-520), Perceval HR (Addgene plasmid \#49082) ${ }^{12}$, or roGFP with the C-terminal anchoring sequence of MAO-B were subcloned into EcoRI and SalI restriction sites of the pFB-TH-SV40 vector and packaged into rAAVs using serotype 9 with titers $2.1-\times 10^{13}$ viral genome copies/ml (Virovek). Mice were anesthetized using an isoflurane precision vaporizer (Smiths Medical PM) and placed in a stereotaxic frame (David Kopf Instruments) with a Cunningham adaptor (Harvard Apparatus) to maintain anesthesia delivery throughout surgery. After exposing the skull, a small hole was drilled and $350 \mathrm{~nL}$ of viral vector delivered via a glass micropipette (Drummond Scientific Company) pulled on a Sutter P-97 puller. The substantia nigra pars compacta (SNc) was targeted at the following coordinates: AP: -3.05 , ML: 1.20, and DV -4.30. All surgeries were performed in wild-type or MAO-A/B knockout mice. Experiments in animals with stereotaxic delivery of AAV viral vectors were performed after at least 10 days post-op.

\section{Ex vivo slice preparation}

Mice were terminally anesthetized with a mixture of ketamine $(50 \mathrm{mg} / \mathrm{kg}) / \mathrm{xylazine}$ $(4.5 \mathrm{mg} / \mathrm{kg})$ and transcardially perfused with ice cold modified artificial cerebrospinal fluid (aCSF) containing in mM: $124.0 \mathrm{NaCl}, 3.0 \mathrm{KCl}, 1.0 \mathrm{CaCl}_{2}, 2.0 \mathrm{MgCl}_{2}, 26 \mathrm{NaHCO}_{3}, 1.0$ 
$\mathrm{NaH}_{2} \mathrm{PO}_{4}$, and 16.66 glucose. Once perfused the brain was rapidly removed and either saggital or coronal slices containing the dorsolateral striatum ( $275 \mu \mathrm{m}$ thick) or the SNc (220 $\mu \mathrm{m}$ thick) sectioned using a vibratome (VT1200S Leica Microsystems). Slices were transferred to a holding chamber containing normal aCSF containing in mM: $124.0 \mathrm{NaCl}$, $3.0 \mathrm{KCl}, 2.0 \mathrm{CaCl}_{2}, 1.0 \mathrm{MgCl}_{2}, 26 \mathrm{NaHCO}_{3}, 1.0 \mathrm{NaH}_{2} \mathrm{PO}_{4}$, and 16.66 glucose and allowed at least 30-40 minutes to recover prior to experiments. All solutions were $\mathrm{pH} 7.4,310-320$ mOsm and continually bubbled with $95 \% \mathrm{O}_{2} / 5 \% \mathrm{CO}_{2}$. Drugs (i.e. methamphetamine and levodopa) used in ex vivo slice experiments were acute, bath application; the duration of application for (methamphetamine 10-20 minutes; levodopa >30 minutes) was far below the duration needed to elicit degeneration which requires $>24$ hour exposure.

\section{Ex vivo redox and ATP measurements}

Oxidant stress was assessed using the redox sensitive roGFP probe targeted to the cytosol, the outer membrane of the mitochondria or the mitochondrial matrix as previously described 11. Slices were transferred to a recording chamber and continuously perfused with normal $\mathrm{aCSF}$ at $32-34^{\circ} \mathrm{C}$. Fluorescence was measured using an Ultima Laser Scanning Microscope system (Bruker) with a DODT contrast detector to provide bright-field transmission images with an Olympus 60/0.9NA lens. A two-photon laser (Chameleon Ultra II, Coherent Inc.) tuned to $920 \mathrm{~nm}$ was used to excite roGFP. Time series image acquisitions of the roGFP probe were obtained with 60 frames obtained over $\sim 20$ seconds with $0.195 \mu \mathrm{m} \times 0.195 \mu \mathrm{m}$ pixels and 10-12 $\mu \mathrm{sec}$ dwell times. The dynamic range of the probe was determined with 2 $\mathrm{mM}$ dithiothreitol, a reducing agent, and $200 \mu \mathrm{M}$ aldrithiol, an oxidizing agent, which were sequentially perfused and $t$-series acquired with each to determine the maximal and minimal fluorescence intensity. Test measurements were calculated as relative oxidation. $T$-series were analyzed offline and fluorescence measurements in multiple regions of interest evaluated with background subtracted ${ }^{21}$. ATP:ADP ratioswere measured using the biosensor Perceval HR ${ }^{12}$. T-series image acquistions of the ATP biosensor were obtained as for roGFP experiments but were obtained over $\sim 10$ seconds with aCSF containing $3.5 \mathrm{mM}$ glucose and slices perfused with $10 \mathrm{mM} \mathrm{KCl}$ or electrical stimulation $(350 \mu \mathrm{A}, 2 \mathrm{~ms})$ to evoke activity. Two $t$-series were obtained for each measurement, one with 950nm excitation wavelength corresponding to ATP and a second at $820 \mathrm{~nm}$ corresponding to ADP ${ }^{12}$. ATP and ADP fluorescence were analyzed offline and quantified as ATP/ADP ratio. Ratiometric measurement was not possible during application of the electrical stimulation trains. As such, 950nm excitation was used to measure ATP binding to the probe. All final data are presented as percent of control (aCSF perfusion) and normalized to minimum ratio measured in nonhydrolyzable 2-deoxyglucose.

\section{Human iPSC culture and neural differentiation}

The iPSC line was generated from skin fibroblasts of a healthy individual through retroviral expression of OCT4, SOX2, cMYC, KLF4 and previously clinically characterized ${ }^{23,24}$. Differentiation of iPSCs into midbrain dopaminergic neurons was done according to published protocols resulting in $\sim 70 \%$ expressing tyrosine hydroxylase with an array of dendritic and axonal processes ${ }^{25}$. To help control neuralization variability, cells were passaged en bloc (size of 1-2 $\mathrm{mm}$ ) between days 11 and 14, followed by plating onto polyd-lysine (PDL)/laminin coated $10 \mathrm{~cm}$ dishes. Between days 25 and 30, neural blocks were 
passed by accutase treatment onto PDL/laminin coated culture dishes. Neuralization growth factors were withdrawn at day 40 and neurons were maintained in Neurobasal media (Life Technologies) containing Neurocult SM1 (Stemcell technologies).

\section{Redox, ATP, and mitochondrial membrane potential measurements in iPSC-derived dopaminergic neurons}

Human iPSC-derived dopaminergic neurons were grown on $12 \mathrm{~mm}$ glass coverslips and imaged between 75 and 88 days post-differentiation. Two to four days before experimentation, dopaminergic neurons were infected with viral vectors targeting roGFP expression to mitochondrial matrix (AAV9-TH-mitoroGFP), mitochondrial intermembrane space (AAV9-TH-IMSroGFP) ${ }^{11}$, or the cytosol (AAV9-TH-cytoroGFP) ${ }^{26}$. Cultures were transferred to an imaging chamber mounted on an inverted epifluorescence microscope (Nikon TE300) with a 40X/1.35NA oil-immersion objective. The imaging chamber was superfused with aCSF at a flow rate of $1 \mathrm{ml} / \mathrm{min}$. Imaging experiments were carried out at $31-33^{\circ}$ C. Cells were illuminated by Polychrome V (TILL Photonics) and emissions were captured with a cooled CCD camera (Hamamatsu ImagEM) and Slidebook imaging software (Intelligent Imaging Innovations). Regions of interests in the dendrites or axon were selected for image analysis. For roGFP redox measurements, cells were exposed at two wavelengths ( 410 and $470 \mathrm{~nm}$ ) sequentially for $50 \mathrm{~ms}$ each, time-lapse ratiometric images (F410/F470) were captured every $30 \mathrm{sec}$. dopaminergic neurons expressing roGFP probes were imaged for $15 \mathrm{~min}$ to establish stable baselines; the perfusion buffer then switched to aCSF containing $100 \mu \mathrm{M}$ levodopa for $30 \mathrm{~min}$, or $10 \mu \mathrm{M}$ rasagiline $/ 5 \mu \mathrm{M}$ clorigyline for 30 min followed by $100 \mu \mathrm{M}$ levodopa $+10 \mu \mathrm{M}$ rasagiline $/ 5 \mu \mathrm{M}$ clorigyline for $30 \mathrm{~min}$. Afterwards, neurons were perfused with $2 \mathrm{mM}$ dithiothreitol for $20 \mathrm{~min}$ and then $100 \mu \mathrm{M}$ aldrithiol for 20 min to obtain full roGFP dynamic range ${ }^{26}$.

For measurement of cytosolic ATP:ADP ratios, dopaminergic neurons expressing Perceval HR were excited at two wavelengths (420 and $490 \mathrm{~nm}$ ) sequentially for $50 \mathrm{~ms}$ each, respective images were captured at $530 \mathrm{~nm}$ every $30 \mathrm{sec}$. Cells were perfused with aCSF containing $3.5 \mathrm{mM}$ glucose. After establishing stable baselines and treatment of $100 \mu \mathrm{M}$ levodopa for $30 \mathrm{~min}$, with or without MAO inhibitors (10 $\mu \mathrm{M}$ rasagiline $/ 5 \mu \mathrm{M}$ clorgyline), cells were perfused with aCSF containing $3.5 \mathrm{mM} 2-\mathrm{DG}$ (replacing glucose) and $10 \mu \mathrm{M}$ oligomycin to obtain the value of minimum ATP/ADP ratio. All final data are presented as percent of control (aCSF perfusion).

To monitor changes in mitochondria membrane potential, dopaminergic neurons were loaded with $20 \mathrm{nM}$ TMRM for 45min, washed, and maintained in $2 \mathrm{nM}$ TMRM during imaging. Cells were exposed at $550 \mathrm{~nm}$ for $50 \mathrm{~ms}$, and time-lapse images were captured every $30 \mathrm{sec}$. After imaging approximately $15 \mathrm{~min}$ to establish stable baselines, complex III inhibitor myxothiazol $(2 \mu \mathrm{M})$ and adenine nucleotide translocase inhibitor carboxyatractyloside $(1 \mu \mathrm{M})$ were added to perfusion buffer for the remainder of the imaging. Thirty minutes later, cells were treated with $100 \mu \mathrm{M}$ levodopa and imaging continued for an additional $30 \mathrm{~min}$; to test MAO inhibition, $10 \mu \mathrm{M}$ rasagiline $/ 5 \mu \mathrm{M}$ clorgyline were added $30 \mathrm{~min}$ prior to $100 \mu \mathrm{M}$ levodopa. Regions of interest with stable baseline fluorescence (less than $15 \%$ variation) were individually fit with centered, second 
order polynomial regressions (average $\mathrm{r}^{2}>0.90$ in all experiments), using data from the thirty minutes following complex III/ANT inhibition (prior to levodopa administration). The mitochondrial polarization index was calculated by dividing observed data by modeled data; deviation of polarization indices from 1.0 indicated deviation from decay trajectory.

\section{Dopamine release with dLight1.3b}

Mice were stereotaxically injected with $350 \mathrm{nl}$ of AAV.CAG.dLight $1.3 \mathrm{~b}$ in the SNc. Ten days following surgery, sagittal slices were prepared as previously described. Image series were taken via 2PLSM. Dopamine release in response to electrical stimulation (350 $\mu \mathrm{A}, 2$ ms pulse width, $1 \mathrm{p}$ or $5 \mathrm{p} 100 \mathrm{~Hz}$ ) was measured. Syanptic blockers (nicotinic acetylcholine receptor antagonist: mecamylamine, D2 receptor antagonist: sulpiride, $10 \mu \mathrm{M}$ ) were used to investigate release probability. ImageJ was used to sum total pixel fluorescence and from these data $\Delta \mathrm{F} / \mathrm{F}_{0}$ and areas under the curve were calculated.

\section{Dopamine release with fast-scan cyclic voltammetry}

Custom built carbon fiber microelectrodes ( 80 - $100 \mu \mathrm{m}$ exposed carbon fiber in silica) were inserted $100 \mu \mathrm{m}$ into the dorsolateral striatum of coronal brain slices, opposite a pronged stimulation electrode. A cyclic waveform sufficient to oxidize and reduce DA ( -0.3 to $1.4 \mathrm{~V}$, $10 \mathrm{~Hz}, 400 \mathrm{~V} / \mathrm{sec}$ ) was applied to the carbon fiber microelectrode (Dagan ChemCLAMP amplifier/headstage driven by Axon Digidata 1440A). Dopamine release was elicited via electrical stimulation (single pulse, $350 \mu \mathrm{A}, 2 \mathrm{~ms}$ ) and measured as current generated at the surface of the carbon fiber microelectrode. Normal stimulation frequency was every 4 minutes, which results in consistent and stable dopamine release. Stimulation frequency was increased to every 10 seconds, which results in dopamine release depletion, to assess the importance of MAO and mitochondrial ATP production to intense energy consumption.

\section{TRAP analysis and RNA sequencing}

Male hemizygous DAT bacTRAP mice ${ }^{22}$ were randomly divided into groups of five mice. Brains were removed and sectioned using an ice-cold Adult Mouse Brain Slicer with $1 \mathrm{~mm}$ coronal slice intervals (Zivic Instruments). From the tissue section containing the midbrain the SNc was dissected under a Nikon SMZ645 light microscope using a 10X lens. Translated mRNAs were purified as described previously ${ }^{27}$. TRAP samples underwent DNase digestion using the RNase-Free DNase Set (Qiagen) and were subsequently purified with the RNeasy MinElute Cleanup Kit (Qiagen). Eluted RNA samples were analyzed on a 2100 Bioanalyzer (Agilent) using RNA Pico Chips (Agilent) to confirm RNA integrity, followed by the measurement of RNA concentrations with the Quant-iT RiboGreen RNA Assay Kit (Life Technologies). cDNAs were prepared with the Ovation RNA-Seq System V2 kit (NuGEN), using an input of 1 ng RNA. 500 ng cDNA from each sample were fragmented on a Covaris S2 Focused Ultrasonicator using the operating conditions recommended by the manufacturer for a target fragment size of $200 \mathrm{bp}$. Fragment size was confirmed on a 2100 Bioanalyzer using High Sensitivity DNA Chips (Agilent). Libraries for RNA sequencing were prepared with the TruSeq RNA Sample Preparation v2 kit (Illumina), starting the manufacturer's low-throughput protocol with the end repair step. The concentration of the RNA-Seq libraries was determined on a 2100 Bioanalyzer using High Sensitivity DNA Chips. Subsequently, two libraries with different adapters were multiplexed 
for sequencing. After confirming the concentration of the multiplexed samples on a 2100 Bioanalyzer using High Sensitivity DNA Chips, samples were analyzed on an Illumina HiSeq 2000 sequencer using $100 \mathrm{bp}$ single-end sequencing. RNA-Seq reads were mapped to the Mus musculus assembly 10 reference genome using TopHat version 2.0.11. FPKM values for all genes in each sample were calculated with Cufflinks version 2.2.1. To analyze differential gene expression between samples, DESeq version 1.14.0 was used under the standard comparison mode.

\section{Statistical analysis}

All data were analyzed in Prism (GraphPad Software La Jolla, CA). Data sets were not assumed to be normal and thus analyzed using non-parametric statistics. Data are presented as box-and-whisker plots depicting median, quartiles, and range. Mann-Whitney (twotailed), Wilcoxon matched-pairs signed rank (two-tailed), and Kruskal-Wallis with Dunns post-hoc analyses were used where appropriate. For clarity, all data are normalized to respective controls and presented as a percent; $\boldsymbol{a}=0.05$. Data collection and analysis were not performed blind to the conditions of the experiments. No statistical methods were used to predetermine sample sizes; sample sizes were determined based on pilot studies and randomization procedures were not used. Additional Information can be found in the Life Sciences Reporting Summary.

\section{Data availability}

Data from this study are available from the corresponding author upon reasonable request.

\section{Code availability}

Analysis routines/code is available from the corresponding author upon reasonable request.

\section{Extended Data}



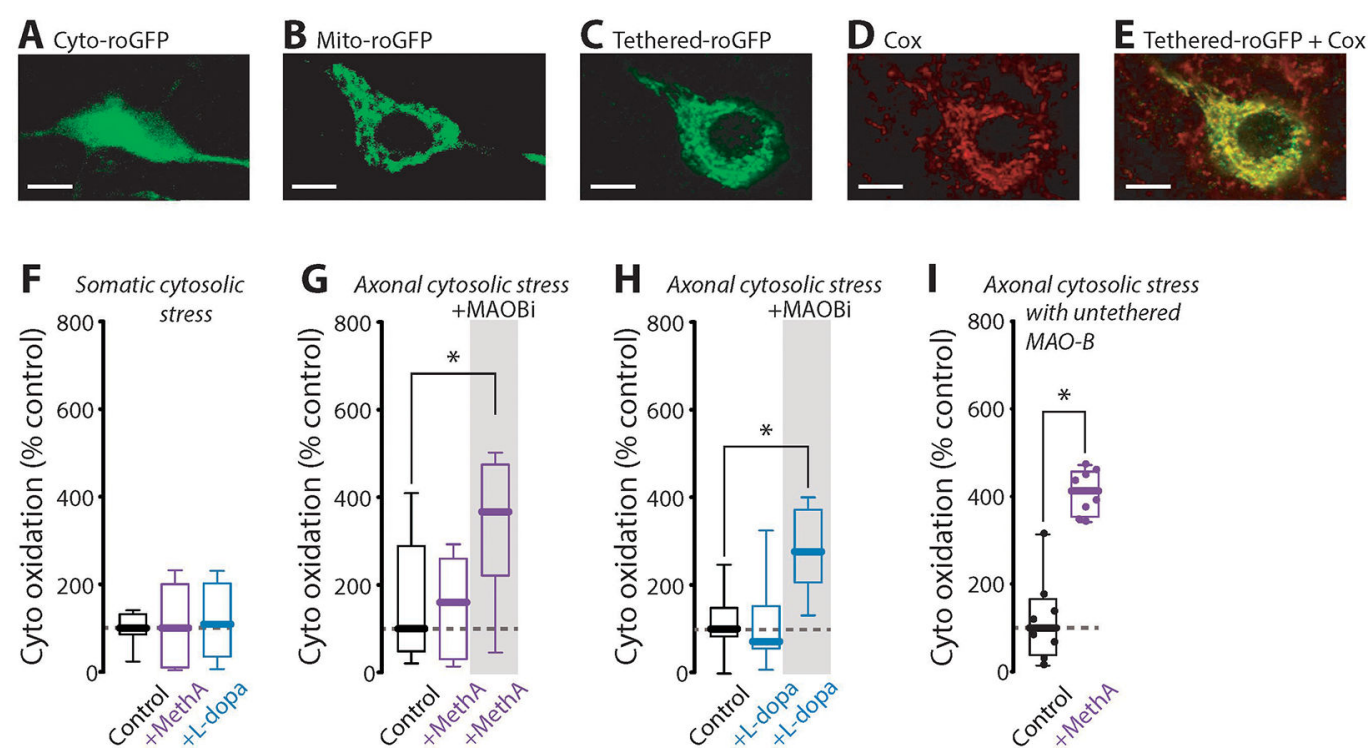

Extended Data Fig. 1. Cytosolic thiol oxidation

The redox sensitive probe roGFP was targeted to the various cellular compartments compartment of substantia nigra pars compacta dopamine neurons for experiments throughout the manuscript. (A) Sample image illustrating the expression pattern of cytoroGFP. (B) Sample image illustrating the expression pattern of Mito-roGFP; note the pattern of the mitochondrial matrix and absence of nuclear localization. (C) Sample image illustrating the expression pattern of roGFP with a targeting sequence localizing the probe to the outter membrane of the mitochondria (same targeting sequence used by monoamine oxidase enzymes); the tethered roGFP presents a similar pattern of distribution of MitoroGFP suggesting mitochondrial localization. (D) Cytochrome c oxidase (Cox) counterstain of the same cell depicted in panel c. (E) Merged image of the tethered-roGFP and Cox counterstain demonstrating colocalizaton. Scale bars denote $20 \mu \mathrm{m}$. dopaminergic neurons and levels of oxidant status were measured. (F) Somatic cytosolic oxidation was unaltered by $10 \mu \mathrm{M}$ methamphetamine (+MethA; $\mathrm{n}=10$ cells/3 mice) or $100 \mu \mathrm{M}$ levodopa (+1-dopa; $\mathrm{n}=11$ cells $/ 3$ mice); control $\mathrm{n}=11$ cells $/ 2$ mice; Kruskal-Wallis test; $\mathrm{p}=0.9154$. Box-andwhisker plots depict median, quartiles, and range. $(\mathrm{G})+$ MethA $(\mathrm{n}=11$ slices/4 mice) had no effect on cytosolic oxidation in axons unless slices were incubated with $10 \mu \mathrm{M}$ of the monoamine oxidase inhibitor rasagiline ( + MAOBi; $n=10$ slices $/ 4$ mice); control $n=11$ slices/3 mice (Kruskal-Wallis test; $\mathrm{p}=0.0108$ ). Box-and-whisker plots depict median, quartiles, and range. $(\mathrm{H})+1$-dopa ( $\mathrm{n}=13$ slices/4mice) had no effect on axonal cytosolic oxidation unless in the presence of $10 \mathrm{uM}+\mathrm{MAOBi}(\mathrm{n}=14$ slices $/ 4$ mice); control $\mathrm{n}=10$ slices/3mice (Kruskal-Wallis test; $\mathrm{p}<0.001$ ). Box-and-whisker plots depict median, quartiles, and range. (I) Monoamine oxidase knockout mice were stereotaxically delivered a variant of MAO-B lacking the tethering sequence targeting it to the outer membrane of the mitochondria and tested for methamphetamine (+Meth; $10 \mu \mathrm{M})$-induced cytosolic thiol oxidation in axons. +Meth increased cytosolic oxidation in slices from mice expressing untethered MAO-B; $n=8$ slices/3 mice (Wilcoxon matched-pairs two-sided signed rank test, $\mathrm{p}=0.0078$ ). Box-and-whisker plots depict median, quartiles, and range. ${ }^{*} \mathrm{p}<0.05$. 


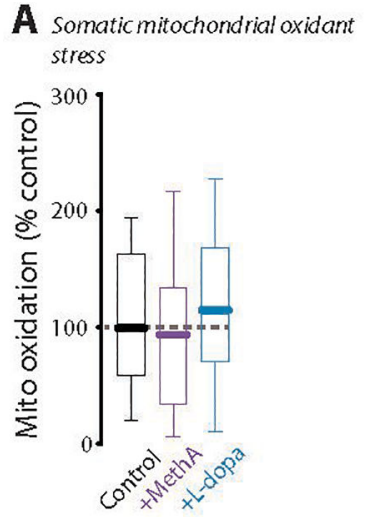

B Axonal mitochondrial oxidant stress
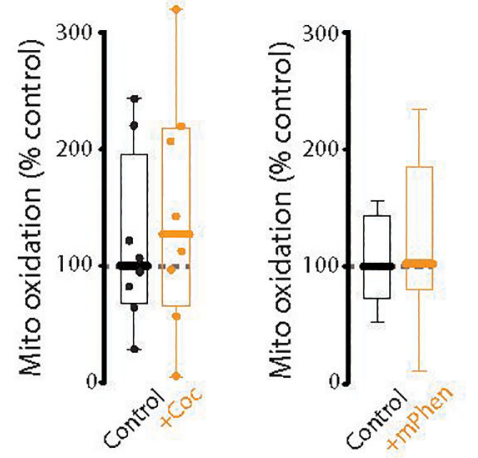

C Axonalmitochondrial oxidant stress

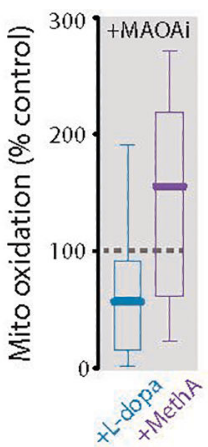

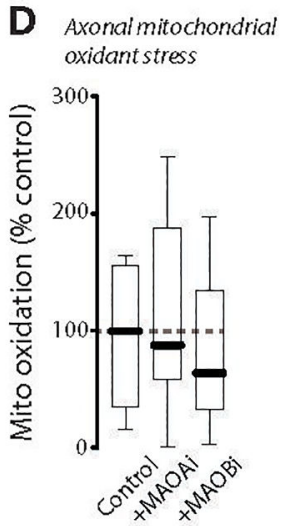

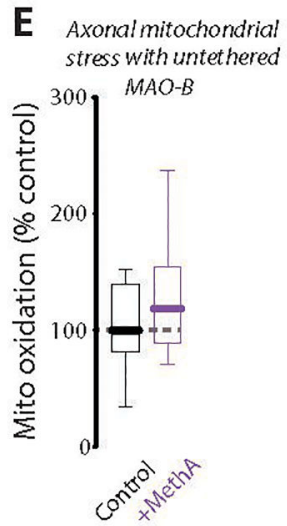

F Axonal mitochondrial oxidant stress in humandopaminergic neurons

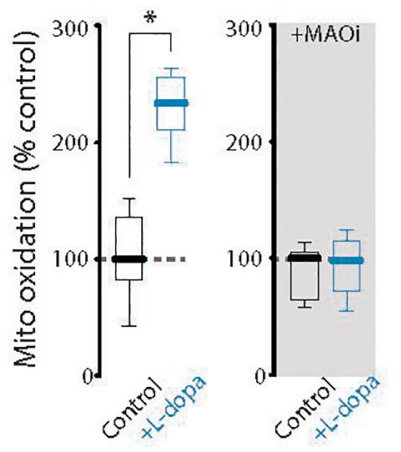

\section{Extended Data Fig. 2. Mitochondrial thiol oxidation}

The redox sensitive probe roGFP was targeted to the mitochondrial matrix of substantia nigra pars compacta dopaminergic neurons and levels of thiol redox status were measured. (A) Somatic Mito-roGFP oxidation was unchanged by treatments; control $(\mathrm{n}=11$ cells/ 6 mice), $10 \mu \mathrm{M}$ methamphetamine (+MethA; $\mathrm{n}=12$ cells/4 mice), or $100 \mu \mathrm{M}$ levodopa (+1dopa; $\mathrm{n}=18$ cells $/ 5$ mice); Kruskal-Wallis test; $\mathrm{p}=0.5341$. Box-and-whisker plots depict median, quartiles, and range. (B) Cocaine (left; $5 \mu \mathrm{M}$; +Coc) had no effect on mitochondrial thiol redox status in substantia nigra pars compacta dopaminergic axons (Wilcoxon matched-pairs signed rank test $\mathrm{p}=0.6454 ; \mathrm{n}=8$ slices $/ 2$ mice. Methylphenidate (right; $5 \mu \mathrm{M}$; + mPhen) also had no effect on axonal Mito-roGFP oxidation status ( $\mathrm{n}=11$ slices $/ 3$ mice); Wilcoxon matched-pairs two-sided signed rank test; $\mathrm{p}=0.4131$. Box-and-whisker plots depict median, quartiles, and range. (C) Clorgyline (5 $\mu \mathrm{M}$; +MAOAi), a monoamine oxidase A inhibitor, prevented $100 \mu \mathrm{M}$ levodopa (+1-dopa; $\mathrm{n}=12$ slices $/ 3$ mice; analyzed with Figure 1E) and $10 \mu \mathrm{M}$ methamphetamine (+MethA; $\mathrm{n}=12$ slices/4 mice; analyzed with Figure 1B)induced axonal Mito-roGFP oxidation. Box-and-whisker plots depict median, quartiles, and range. (D) Monoamine oxidase inhibitors alone had no effect on axonal mitochondrial thiol redox status; control $n=10$ slices $/ 3$ mice, + MAOAi $n=11$ slices $/ 3$ mice, + MAOBi $n=11$ slices/3 mice (Kruskal-Wallis test; $\mathrm{p}=0.7413$ ). Box-and-whisker plots depict median, quartiles, and range. (E) Monoamine oxidase knockout mice were stereotaxically delivered a variant of MAO-B lacking the tethering sequence targeting it to the outer membrane of the mitochondria and tested for methamphetamine (+Meth; $10 \mu \mathrm{M})$-induced mitochondrial thiol 
oxidation in axons. +Meth had no effect on mitochondrial oxidant staus in slices from mice expressing untethered MAO-B; $\mathrm{n}=12$ slices/3 mice (Wilcoxon matched-pairs two-sided signed rank test, $\mathrm{p}=0.2036$ ). Box-and-whisker plots depict median, quartiles, and range. (F) Human iPSC-derived dopaminergic neurons were transfected with the redox sensitive probe roGFP targeted to the mitochondrial matrix. Basal levels of oxidant status were measured (control) followed by incubation with $100 \mu \mathrm{M}$ levodopa (+l-dopa). +l-dopa increased axonal mitochondrial thiol oxidation; experiment performed using a within-subject design with repeated measures (left; $\mathrm{n}=15$ axons; Wilcoxon matched-pairs two-sided signed rank test; $\mathrm{p}<0.0001$ ). In a separate set of cells monoamine oxidase inhibition (right) with $5 \mu \mathrm{M}$ clorgyline $+10 \mu \mathrm{M}$ rasagiline (+MAOi) prevented +l-dopa-induced axonal mitochondrial thiol oxidation; experiment performed using a within-subject design with repeated measures; Wilcoxon matched-pairs two-sided signed rank test; $\mathrm{p}=0.4973$ ); $\mathrm{n}=14$ axons; box-andwhisker plots depict median, quartiles, and range. ${ }^{*} \mathrm{p}<0.05$. 

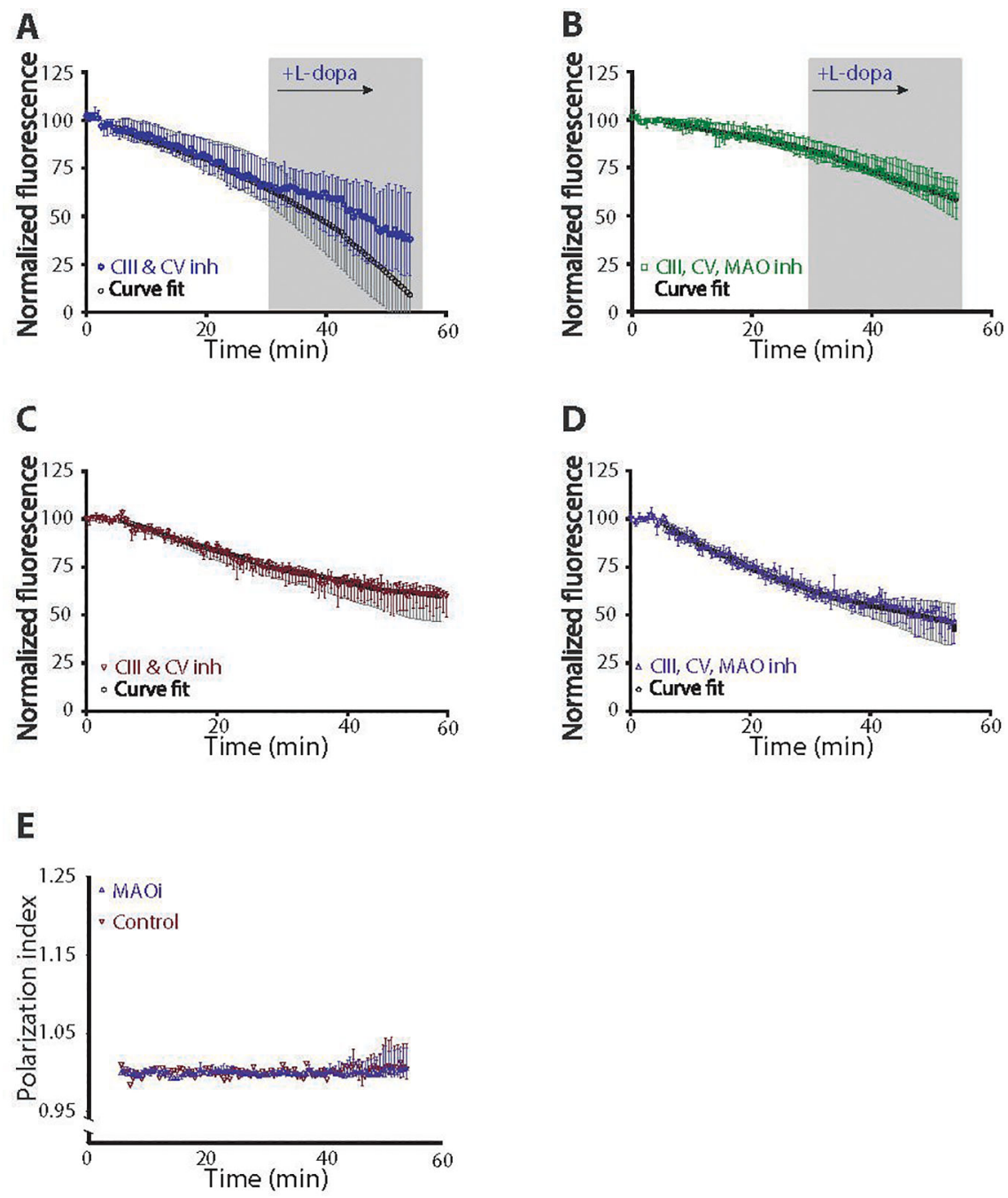

\section{Extended Data Fig. 3. Mitochondrial membrane potential measured by TMRM}

(A) An image of a dopamine (DA) differentiated neuron expressing the ATP biosensor Perceval HR (upper left) and high magnification highlighting an axonal segment (lower right); scale bars denote $10 \mu \mathrm{m}$. (B) Sample traces (left) illustrating $100 \mu \mathrm{M}$ levodopa (+Ldopa)-induced increase in ATP/ADP ratio. $+\mathrm{L}$-dopa increased axonal ATP synthesis measured as ATP/ADP ratio compared to control (middle; $\mathrm{n}=15$ axons; Wilcoxon matchedpairs two-sided signed rank test; $\mathrm{p}<0.0001$ ). In a separate set of cells $+\mathrm{L}$-dopa-induced ATP synthesis was prevented by MAO inhibition with $5 \mu \mathrm{M}$ clorgyline $+10 \mu \mathrm{M}$ rasagiline (right; MAOi; $n=15$ axons; Wilcoxon matched-pairs two-sided signed rank test; $\mathrm{p}=0.0554$ ); experiments performed using a within-subject design with repeated measures. Box-andwhisker plots depict median, quartiles, and range. (C) The ATP biosensor Perceval HR expresses throughout dopaminergic neurons as evidenced by sample images in the dorsolateral striatum; low (upper left; scale bar denotes $500 \mu \mathrm{m}$ ) and high magnification images (lower right; scale bar denotes $10 \mu \mathrm{m}$ ) illustrating striatal expression of Perceval HR in SNc DA axons. (D) +L-dopa increased axonal ATP synthesis measured as ATP/ADP ratio in $\mathrm{SNc}$ axons in the dorsolateral striatum compared to control (middle; $\mathrm{n}=8$ slices $/ 3$ mice; Wilcoxon matched-pairs two-sided signed rank test; $\mathrm{p}=0.0078$ ). In a separate set of ex vivo 
slices, +L-dopa-induced ATP synthesis was prevented by MAO inhibition with $10 \mu \mathrm{M}$ rasagiline (right; +MAOBi; $\mathrm{n}=9$ slices/2 mice; Wilcoxon matched-pairs two-sided signed rank test; $p=0.2031$ ); experiment performed using a within-subject design with repeated measures. Box-and-whisker plots depict median, quartiles, and range. (E) Cartoon illustrating the bioenergetically demanding process of DA release, reuptake, and packaging into vesicles. (F) Electrical stimulation trains $(0.1 \mathrm{~Hz})$ were used to deplete axonal ATP. Repeated measurements were taken at $950 \mathrm{~nm}$ and presented as $\Delta \mathrm{F} / \mathrm{F}_{0}$; repetitive stimulation decreased axonal ATP. In the presence of the MAO-B inhibitior rasagiline (+MAO-Bi) ATP levels were further decreased and yet further with complex V inhibition (+oligomycin) (lines are median values, shading is interquartile range; control $n=8,+$ MAOBi $n=9$, and +oligomycin $n=6$ brain slices). (G) To better visualize the contribution of MAO-B to ATP generation, the measurements in the presence of rasagaline were subtracted from those in control aCSF to yield MAO-B dependent ATP (line represents the median MAO-B/aCSF differential, shading is interquartile range; $\mathrm{n}=9$ brain slices). (H) Pre-train: Inhibition of MAO-B or complex V (+oligomycin) both decreased the ATP:ADP bioenergetic index (Kruskal-Wallis test: aCSF vs rasagaline $\mathrm{p}=0.011$, aCSF vs oligomycin $\mathrm{p}=0.0005$; control $\mathrm{n}=7$; +MAOBi $\mathrm{n}=7$; +oligomycin $\mathrm{n}=6$ brain slices). Post-train: Electrical stimulation trains decreased ATP signal in all cases but the effect was more pronounced in the absence of mitochondrial ATP production (Kruskal-Wallis test: aCSF vs oligomycin $\mathrm{p}=0.0005$, control $\mathrm{n}=6$; +MAOBi $\mathrm{n}=6$, +oligomycin $\mathrm{n}=6$ ). Box-and-whisker plots depict median, quartiles, and range(I) Images of dopamine axons expressing dopamine biosensor dLight $1.3 \mathrm{~b}$ before and after electrical stimulation (1p, $350 \mu \mathrm{A}, 2 \mathrm{~ms})$; scale bar denotes $10 \mu \mathrm{M}$. (J) Release probability was interrogated using electrical stimulation to mimic tonic $(1 \mathrm{p}, 2 \mathrm{~ms}, 350 \mu \mathrm{A})$ or phasic dopamine release $(5 \mathrm{p} 100 \mathrm{~Hz}, 2 \mathrm{~ms}, 350 \mathrm{uA})$ in the presence of synaptic blockade (10 $\mu \mathrm{M}$ mecamylamine, $10 \mu \mathrm{M}$ sulpiride). Traces of quantified dLight fluorescence in response to tonic or phasic release stimulation. Line: median, shading $95 \% \mathrm{CI}$; control $\mathrm{n}=5$, + MAOBi $n=6$, and +oligomycin $n=4$ brain slices. (K) No statistically significant difference was seen in tonic firing in response to $+\mathrm{MAOBi}$ (rasagaline, $10 \mathrm{uM}$ ) or +oligomycin (10 $\mathrm{uM})$. Phasic firing was significantly decreased by $+\mathrm{MAOBi}$ and further decreased by +oligomycin (2-way ANOVA, $5 \mathrm{p} 100 \mathrm{~Hz}$ : control vs rasagaline $\mathrm{p}=0.015$, control vs oligomycin $\mathrm{p}<0.0001$, rasagaline vs oligomycin $\mathrm{p}=0.028$; control $\mathrm{n}=5,+\mathrm{MAOBi} \mathrm{n}=6$, and +oligomycin $\mathrm{n}=4$ brain slices). Stimulation increases release $(\mathrm{F}=169.63, \mathrm{DFd}=12$, $\mathrm{p}<0.0001)$. Drug affects release $(\mathrm{F}=10.07, \mathrm{DFd}=12, \mathrm{p}=0.003$. The drugs alter stimulus response $(\mathrm{F}=5.64, \mathrm{DFd}=12, \mathrm{p}=0.019)$. Box-and-whisker plots depict median, quartiles, and range. $* \mathrm{p}<0.05$. 

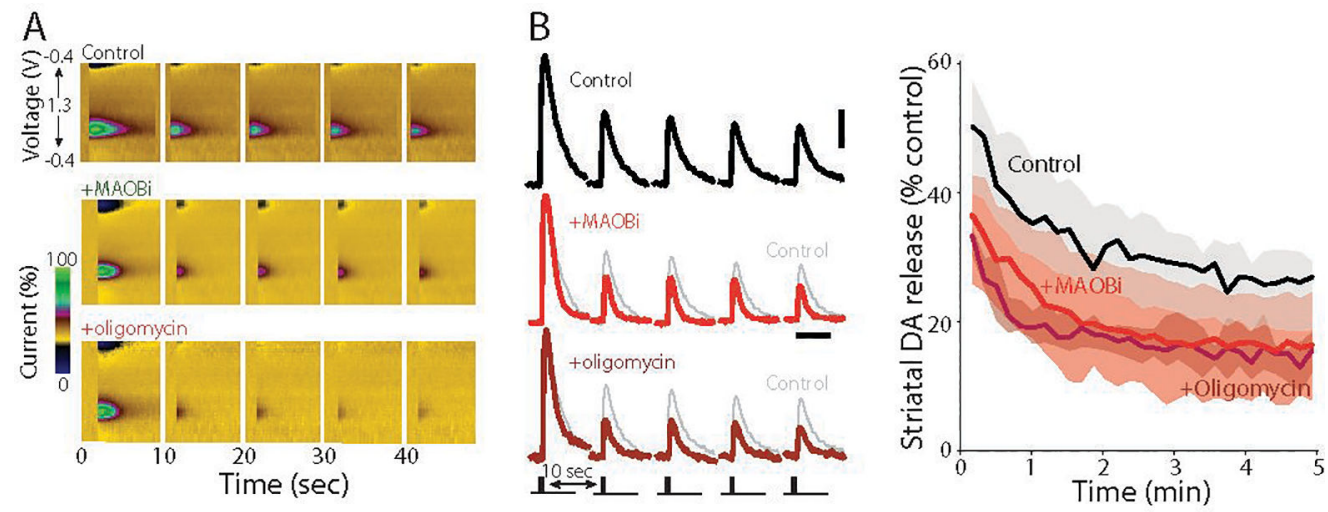

Extended Data Fig. 4. MAO is necessary for maintaining phasic dopamine release

(A) Sample color plots depicting the effects of MAO inhibition (10 $\mu \mathrm{M}$ rasagiline; $+\mathrm{MAOBi})$ and ATP synthase inhibition with oligomycin $(10 \mu \mathrm{M})$ on repeated DA release using fast scan cyclic voltammetry. (B) Summary data of peak DA levels shows increased depletion when MAO and ATP synthesis are inhibited; vertical and horizongal scale bars denote $25 \%$ maximal release and 2.5 seconds, respectively. Data are normalized to the first peak of the stimulation train (line: median, shaded: $95 \%$ confidence interval, control $n=10$, + MAOBi $n=6$, and +oligomycin $n=7$ brain slices, fit with one-phase decay with comparison of fit, $\mathrm{p}<0.001)$.

\section{Acknowledgements}

This study was supported by grants from the JPB Foundation, the IDP Foundation, MJFF and NIH (NS047085) to DJS; an NIH grant (NS076054) to DK; an NIH grant (DA039253) and Northwestern Memorial Foundation grant to SMG; the Boyd and Elsi Welin Professorship and Tsai Family Fund to JCS; NIH grants U01NS103522, U01NS090604, and DPMH107056 to LT. The authors wish to thank the Northwestern Center for Advanced Microscopy (supported by NCI CCSG P30 CA060553) for assistance.

\section{REFERENCES}

1. Segura-Aguilar J et al. Protective and toxic roles of dopamine in Parkinson's disease. J Neurochem 129, 898-915, doi:10.1111/jnc.12686 (2014). [PubMed: 24548101]

2. Fahn S \& Cohen G The oxidant stress hypothesis in Parkinson's disease: evidence supporting it. Ann Neurol 32, 804-812, doi:10.1002/ana.410320616 (1992). [PubMed: 1471873]

3. Kaludercic N, Deshwal S \& Di Lisa F Reactive oxygen species and redox compartmentalization. Front Physiol 5, 285, doi:10.3389/fphys.2014.00285 (2014). [PubMed: 25161621]

4. Dooley CT et al. Imaging dynamic redox changes in mammalian cells with green fluorescent protein indicators. J Biol Chem 279, 22284-22293, doi:10.1074/jbc.M312847200 (2004). [PubMed: 14985369]

5. Sulzer D, Sonders MS, Poulsen NW \& Galli A Mechanisms of neurotransmitter release by amphetamines: a review. Prog Neurobiol 75, 406-433, doi:10.1016/j.pneurobio.2005.04.003 (2005). [PubMed: 15955613]

6. Mosharov EV, Gong LW, Khanna B, Sulzer D \& Lindau M Intracellular patch electrochemistry: regulation of cytosolic catecholamines in chromaffin cells. J Neurosci 23, 5835-5845 (2003). [PubMed: 12843288]

7. Mosharov EV et al. Interplay between cytosolic dopamine, calcium, and alpha-synuclein causes selective death of substantia nigra neurons. Neuron 62, 218-229, doi:10.1016/j.neuron.2009.01.033 (2009). [PubMed: 19409267] 
8. Woodard CM et al. iPSC-derived dopamine neurons reveal differences between monozygotic twins discordant for Parkinson's disease. Cell Rep 9, 1173-1182, doi:10.1016/j.celrep.2014.10.023 (2014). [PubMed: 25456120]

9. Chen K, Holschneider DP, Wu W, Rebrin I \& Shih JC A spontaneous point mutation produces monoamine oxidase A/B knock-out mice with greatly elevated monoamines and anxiety-like behavior. J Biol Chem 279, 39645-39652, doi:10.1074/jbc.M405550200 (2004). [PubMed: 15272015]

10. Brand MD Mitochondrial generation of superoxide and hydrogen peroxide as the source of mitochondrial redox signaling. Free Radic Biol Med 100, 14-31, doi:10.1016/ j.freeradbiomed.2016.04.001 (2016). [PubMed: 27085844]

11. Sabharwal SS, Waypa GB, Marks JD \& Schumacker PT Peroxiredoxin-5 targeted to the mitochondrial intermembrane space attenuates hypoxia-induced reactive oxygen species signalling. Biochem J 456, 337-346, doi:10.1042/BJ20130740 (2013). [PubMed: 24044889]

12. Tantama M, Martinez-Francois JR, Mongeon R \& Yellen G Imaging energy status in live cells with a fluorescent biosensor of the intracellular ATP-to-ADP ratio. Nat Commun 4, 2550, doi:10.1038/ ncomms3550 (2013). [PubMed: 24096541]

13. Youdim MB, Edmondson D \& Tipton KF The therapeutic potential of monoamine oxidase inhibitors. Nat Rev Neurosci 7, 295-309, doi:10.1038/nrn1883 (2006). [PubMed: 16552415]

14. Wimalasena K Vesicular monoamine transporters: structure-function, pharmacology, and medicinal chemistry. Med Res Rev 31, 483-519, doi:10.1002/med.20187 (2011). [PubMed: 20135628]

15. Patriarchi $T$ et al. Ultrafast neuronal imaging of dopamine dynamics with designed genetically encoded sensors. Science 360, doi:10.1126/science.aat4422 (2018).

16. Ashrafi G \& Ryan TA Glucose metabolism in nerve terminals. Curr Opin Neurobiol 45, 156-161, doi:10.1016/j.conb.2017.03.007 (2017). [PubMed: 28605677]

17. Schultz W Multiple dopamine functions at different time courses. Annu Rev Neurosci 30, 259 288, doi:10.1146/annurev.neuro.28.061604.135722 (2007). [PubMed: 17600522]

18. Schultz W Reward functions of the basal ganglia. J Neural Transm (Vienna) 123, 679-693, doi:10.1007/s00702-016-1510-0 (2016). [PubMed: 26838982]

19. Tsai HC et al. Phasic firing in dopaminergic neurons is sufficient for behavioral conditioning. Science 324, 1080-1084, doi:10.1126/science.1168878 (2009). [PubMed: 19389999]

20. Gerfen CR \& Surmeier DJ Modulation of striatal projection systems by dopamine. Annu Rev Neurosci 34, 441-466, doi:10.1146/annurev-neuro-061010-113641 (2011). [PubMed: 21469956]

\section{Methods-only references}

21. Guzman JN et al. Oxidant stress evoked by pacemaking in dopaminergic neurons is attenuated by DJ-1. Nature 468, 696-700, doi:10.1038/nature09536 (2010). [PubMed: 21068725]

22. Brichta $L$ et al. Identification of neurodegenerative factors using translatome-regulatory network analysis. Nat Neurosci 18, 1325-1333, doi:10.1038/nn.4070 (2015). [PubMed: 26214373]

23. Mazzulli JR et al. Gaucher disease glucocerebrosidase and alpha-synuclein form a bidirectional pathogenic loop in synucleinopathies. Cell 146, 37-52, doi:10.1016/j.cell.2011.06.001 (2011). [PubMed: 21700325]

24. Cooper $\mathrm{O}$ et al. Pharmacological rescue of mitochondrial deficits in iPSC-derived neural cells from patients with familial Parkinson's disease. Sci Transl Med 4, 141ra190, doi:10.1126/ scitranslmed.3003985 (2012).

25. Burbulla LF et al. Dopamine oxidation mediates mitochondrial and lysosomal dysfunction in Parkinson's disease. Science 357, 1255-1261, doi:10.1126/science.aam9080 (2017). [PubMed: 28882997]

26. Dryanovski DI et al. Calcium entry and alpha-synuclein inclusions elevate dendritic mitochondrial oxidant stress in dopaminergic neurons. J Neurosci 33, 10154-10164, doi:10.1523/ JNEUROSCI.5311-12.2013 (2013). [PubMed: 23761910]

27. Heiman M, Kulicke R, Fenster RJ, Greengard P \& Heintz N Cell type-specific mRNA purification by translating ribosome affinity purification (TRAP). Nat Protoc 9, 1282-1291, doi:10.1038/ nprot.2014.085 (2014). [PubMed: 24810037] 
A

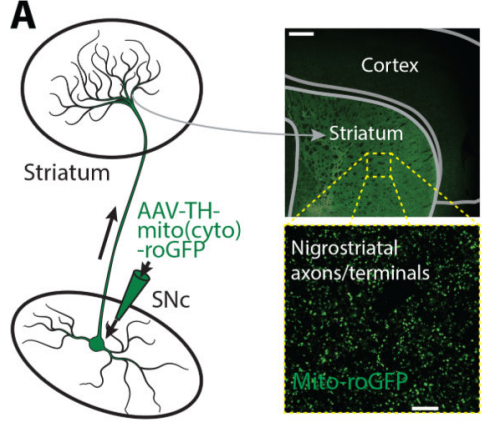

B

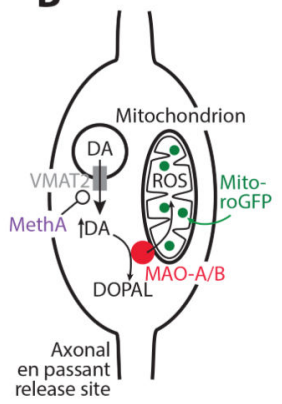

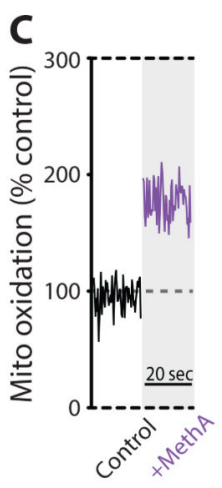

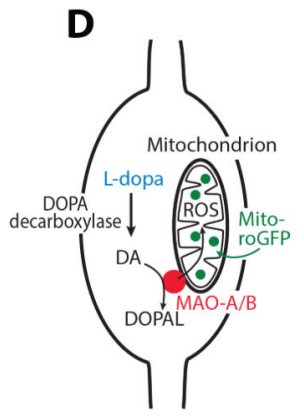

$\mathbf{E}$

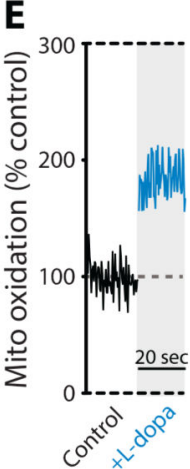

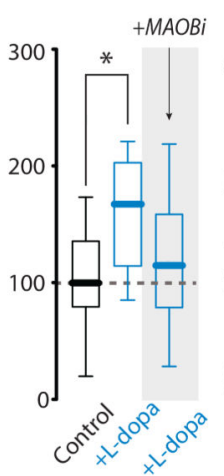

F Reserpinized

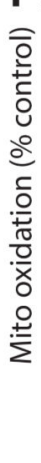

G

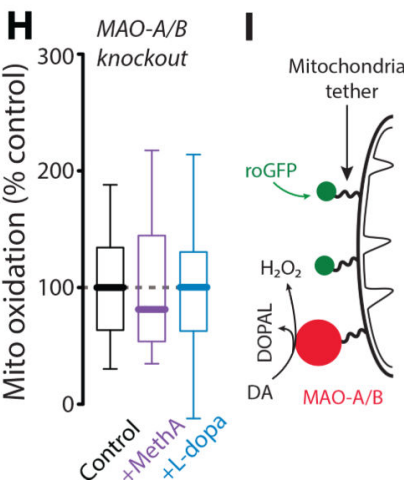

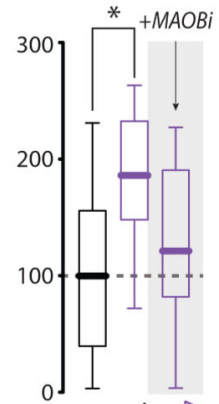

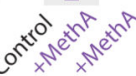

Figure 1. Mitochondrial thiol oxidation is increased by elevating cytosolic dopamine and prevented by inhibiting monoamine oxidase enzymes in ex vivo brain slices.

(A) Cartoon (left) depicting viral delivery of the redox sensitive probe roGFP into dopamine neurons of the substantia nigra pars compacta $(\mathrm{SNc})$. After viral delivery the roGFP probe expresses throughout dopamine neurons (soma, dendrites, and axons); sample images in the dorsolateral striatum (right) with roGFP (targeted to mitochondrial matrix; Mito-roGFP) being expressed in dopaminergic axons. Low (upper left; scale bar denotes $500 \mu \mathrm{m}$ ) and high magnification images (lower right; scale bar denotes $10 \mu \mathrm{m}$ ) illustrating striatal expression of roGFP. (B) Cartoon depicting the actions of methamphetamine (MethA) on vesicular monoamine transporter 2 (VMAT2) in axonal en passant release sites of dopaminergic neurons. (C) MethA (+MethA) increased Mito-roGFP oxidation in dopaminergic axons; sample traces (left) illustrating meth-induced effects on mitochondrial redox compared to control. Perfusion of $10 \mu \mathrm{M}$ MethA (+MethA; $\mathrm{n}=18$ slices/9 mice) increased axonal mitochondrial oxidation (right) relative to control ( $\mathrm{n}=19$ slices/10 mice) and was prevented by $10 \mu \mathrm{M}$ rasagaline, a monoamine oxidase $\mathrm{B}$ inhibitor (+MAOBi; $\mathrm{n}=18$ slices $/ 6$ mice); Kruskal-Wallis test; $\mathrm{p}=0.0043$. Box-and-whisker plots depict median, quartiles, and range. (D) Cartoon depicting levodopa (L-dopa) effects on cytosolic DA and mitochondrial redox in dopaminergic axonal en passant release sites. (E) $+\mathrm{L}-$ dopa $(100 \mu \mathrm{M})$ increased mitochondrial oxidation in dopaminergic axons; sample traces (left) illustrating $+\mathrm{L}-$ dopainduced effects on mitochondrial redox compared to control. Similar to +MethA, +L-dopa ( $\mathrm{n}=20$ slices/6 mice) increased axonal Mito-roGFP oxidation (right) relative to control ( $\mathrm{n}=14$ slices/4 mice) and was prevented by +MAOBi; +L-dopa ( $\mathrm{n}=20$ slices/ 6 mice); KruskalWallis test; $\mathrm{p}=0.0001$. Box-and-whisker plots depict median, quartiles, and range. $(\mathrm{F})$ +MethA $(10 \mu \mathrm{M})$ had no effect on axonal mitochondrial oxidation in slices from mice depleted of dopamine via repeated in vivo administration of reserpine; $n=7$ slices $/ 6$ mice 
(Wilcoxon matched-pairs two-sided test; $\mathrm{p}=0.8048$ ). Box-and-whisker plots depict median, quartiles, and range. (G) Cartoon illustrating MAO-A/B knockout scenario. (H) Neither +MethA $(10 \mu \mathrm{M})$ nor $+\mathrm{L}-$ dopa $(100 \mu \mathrm{M})$ had any effect on axonal mitochondrial redox status in mice lacking both MAO-A and MAO-B; control $n=13$ slices $/ 3$ mice, + MethA $n=11$ slices $/ 3$ mice, $+\mathrm{L}$-dopa $\mathrm{n}=13$ slices $/ 3$ mice (Kruskal-Wallis test; $\mathrm{p}=0.6718$ ). Box-andwhisker plots depict median, quartiles, and range. (I) Cartoon illustrating roGFP tethered to the outer membrane of the mitochondria and the hypothesis that regional $\mathrm{H}_{2} \mathrm{O}_{2}$ production could be produced by MAO metabolism of DA. (J) +MethA $(10 \mu \mathrm{M})$ had no effect on stress at the outer membrane but stress was increased in the presence of $+\mathrm{MAOBi}$; Control $n=12$ slices $/ 3$ mice, + MethA $n=10$ slices $/ 2$ mice, and +MethA +MAOBi $n=11$ slices $/ 3$ mice; Kruskal-Wallis test; $\mathrm{p}=0.0198$. Box-and-whisker plots depict median, quartiles, and range. $* \mathrm{p}<0.05$. 


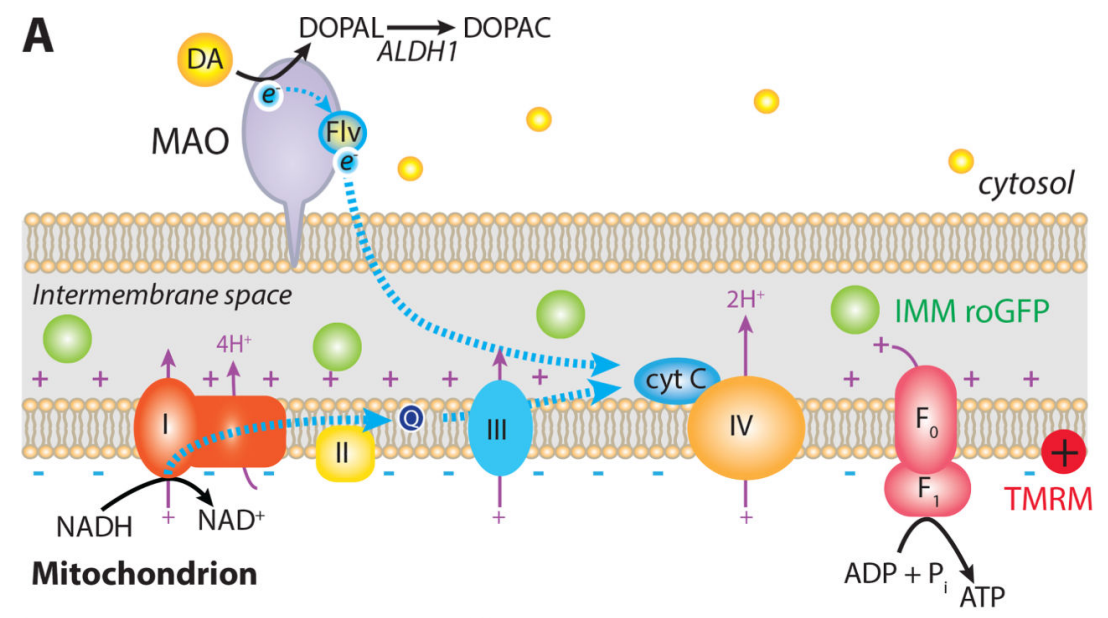

B

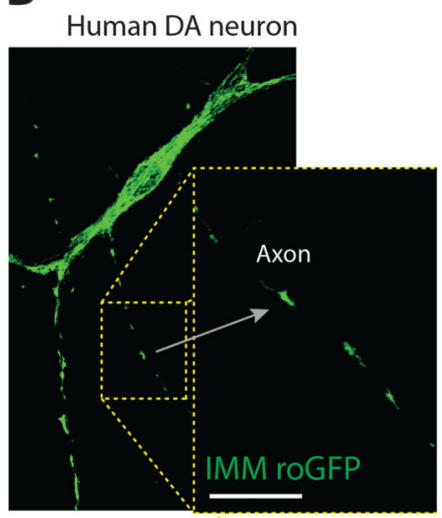

Axonal mitochondrial oxidant stress

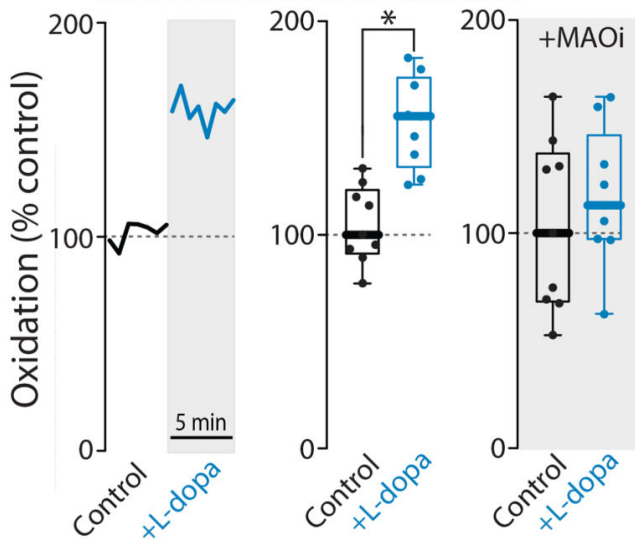

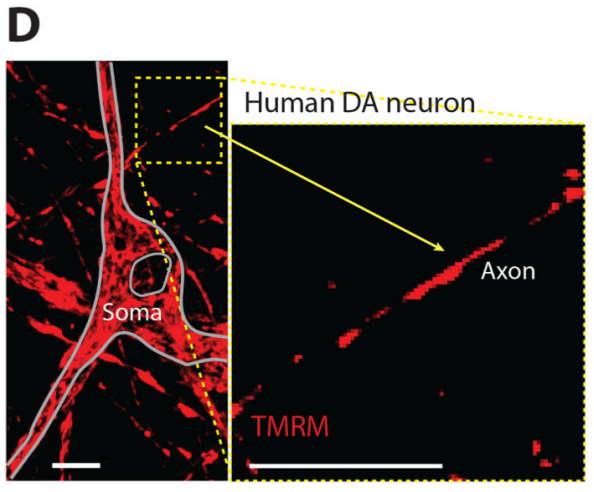

E Axonal mitochondrial $\Delta \psi_{m}$

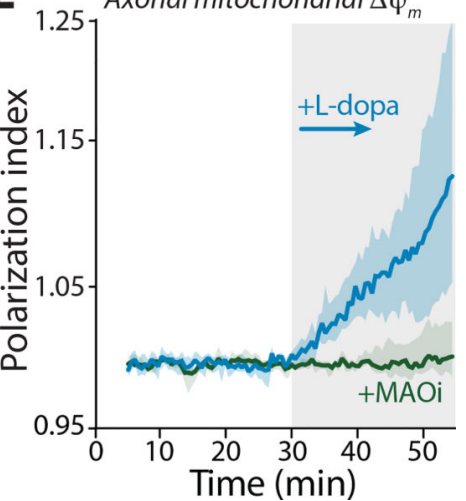

Figure 2. Elevating cytosolic dopamine with levodopa increased mitochondrial thiol oxidation by transferring electrons to the intermembrane space in human iPSC-derived dopamine neurons.

(A) Cartoon depicting the transfer of electrons from monoamine oxidase (MAO) metabolism of dopamine (DA) to mitochondrial intermembrane space. (B) Image of a dopamine differentiated neuron expressing the redox sensitive probe roGFP in the mitochondrial intermembrane space (left) and high magnification highlighting an axonal segment (right); scale bars denote $10 \mu \mathrm{m}$. (C) Sample traces (left) illustrating $100 \mu \mathrm{M}$ levodopa (+L-dopa)induced increase in intermembrane thiol oxidation relative to control. + L-dopa increased axonal oxidation in the intermembrane space compared to control; experiment performed 
using a within-subject design with repeated measures (middle; $\mathrm{n}=9$ axons; Wilcoxon matched-pairs two-sided signed rank test; $\mathrm{p}=0.0039$ ). In a separate set of cells, +L-dopainduced oxidation was prevented by MAO inhibition with $5 \mu \mathrm{M}$ clorgyline $+10 \mu \mathrm{M}$ rasagiline (right); experiment performed using a within-subject design with repeated measures ( MAOi; $n=9$ axons; Wilcoxon matched-pairs signed rank test; $\mathrm{p}=0.25$ ). Box-andwhisker plots depict median, quartiles, and range. (D) Mitochondrial membrane potential was measured with TMRM to demonstrate the transfer of electrons to mitochondria by MAO metabolism of DA; sample one-photon image of a dopamine-differentiated neuron loaded with TMRM dye (upper left) and high magnification image highlighting an axonal segment (lower right); scale bars denote $10 \mu \mathrm{m}$. (E) Addition of $2 \mu \mathrm{M}$ myxothiazol to inhibit complex III and $1 \mu \mathrm{M}$ carboxyatractyloside to inhibit adenine nucleotide translocase resulted in fluorescent decay of the TMRM signal. This decay was fit mathematically with a polynomial to characterize baseline decay (Extended Data Figure 3). The observed data was normalized to the decay fit, generating a polarization index, with deviations from 1.0 indicating deviation from the fitting equation. Perfusion with $100 \mu \mathrm{M}$ levodopa (+1-dopa; $\mathrm{n}=14$ axons) caused a significant deviation and was prevented by MAOi ( $\mathrm{n}=9$ axons); data presented as median \pm quartiles. Data were analyzed by comparison of fit of a quadratic, constrained to the time point of levodopa administration $(\mathrm{p}<0.0001)$. 

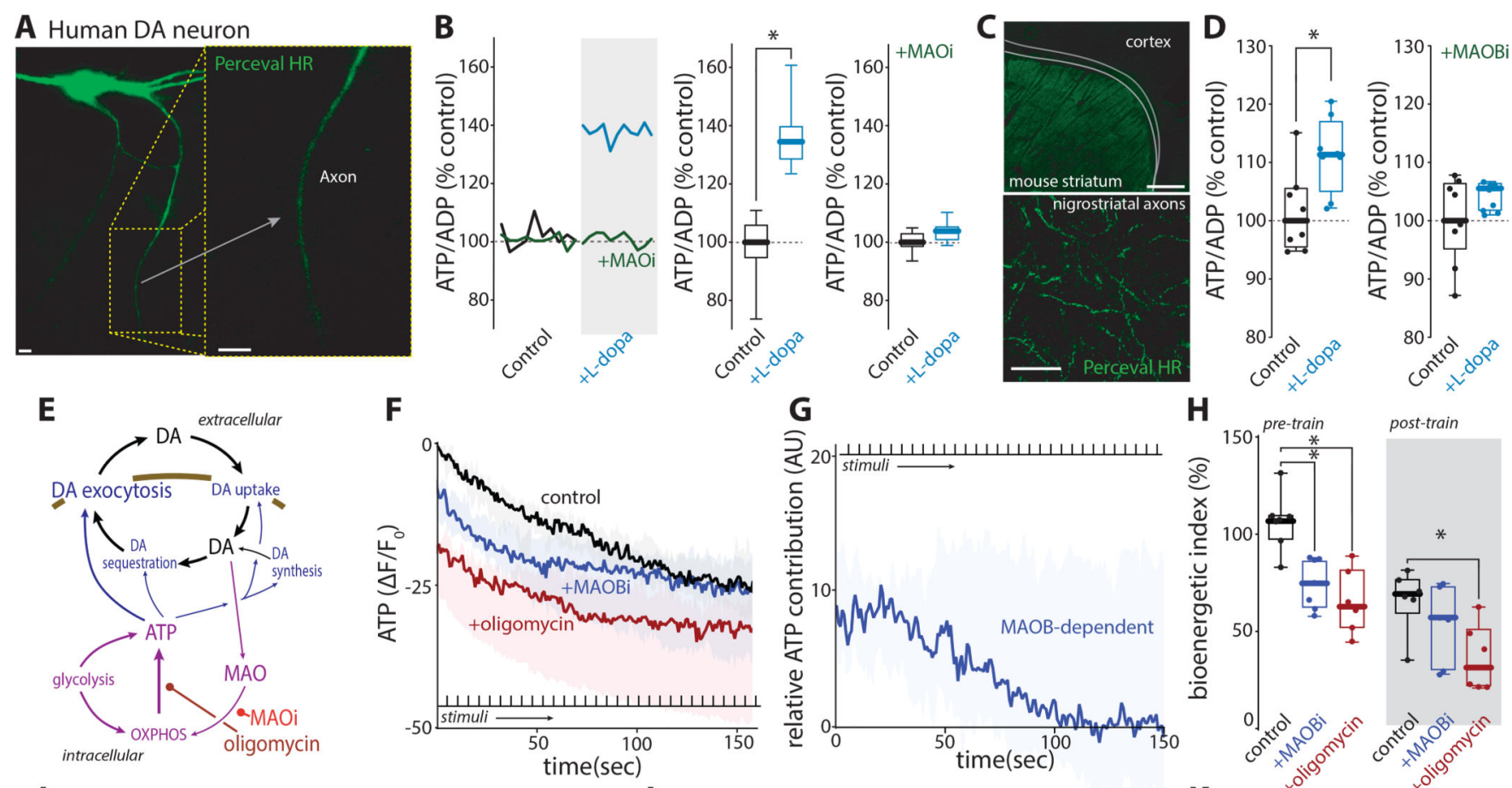

H

I
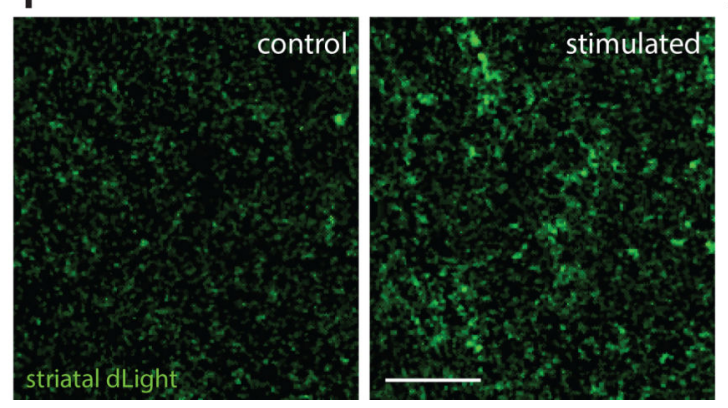

J

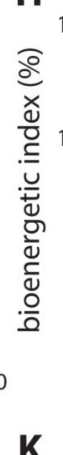

post-train
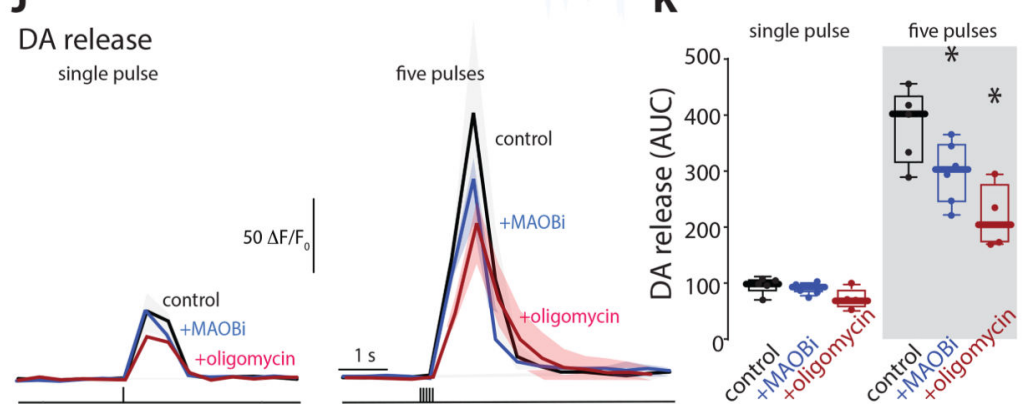

Figure 3. Elevating cytosolic dopamine with levodopa increased ATP synthesis and was necessary for phasic dopamine release.

(A) An image of a dopamine (DA) differentiated neuron expressing the ATP biosensor Perceval HR (upper left) and high magnification highlighting an axonal segment (lower right); scale bars denote $10 \mu \mathrm{m}$. (B) Sample traces (left) illustrating $100 \mu \mathrm{M}$ levodopa (+Ldopa)-induced increase in ATP/ADP ratio. +L-dopa increased axonal ATP synthesis measured as ATP/ADP ratio compared to control (middle; $\mathrm{n}=15$ axons; Wilcoxon matchedpairs two-sided signed rank test; $\mathrm{p}<0.0001)$. In a separate set of cells + L-dopa-induced ATP synthesis was prevented by MAO inhibition with $5 \mu \mathrm{M}$ clorgyline $+10 \mu \mathrm{M}$ rasagiline (right; MAOi; $n=15$ axons; Wilcoxon matched-pairs two-sided signed rank test; $\mathrm{p}=0.0554$ ); experiments performed using a within-subject design with repeated measures. Box-andwhisker plots depict median, quartiles, and range. (C) The ATP biosensor Perceval HR expresses throughout dopaminergic neurons as evidenced by sample images in the dorsolateral striatum; low (upper left; scale bar denotes $500 \mu \mathrm{m}$ ) and high magnification images (lower right; scale bar denotes $10 \mu \mathrm{m}$ ) illustrating striatal expression of Perceval HR in SNc DA axons. (D) +L-dopa increased axonal ATP synthesis measured as ATP/ADP ratio in $\mathrm{SNc}$ axons in the dorsolateral striatum compared to control (middle; $\mathrm{n}=8$ slices/3 mice; 
Wilcoxon matched-pairs two-sided signed rank test; $\mathrm{p}=0.0078$ ). In a separate set of ex vivo slices, $+\mathrm{L}$-dopa-induced ATP synthesis was prevented by MAO inhibition with $10 \mu \mathrm{M}$ rasagiline (right; +MAOBi; $\mathrm{n}=9$ slices/2 mice; Wilcoxon matched-pairs two-sided signed rank test; $\mathrm{p}=0.2031$ ); experiment performed using a within-subject design with repeated measures. Box-and-whisker plots depict median, quartiles, and range. (E) Cartoon illustrating the bioenergetically demanding process of DA release, reuptake, and packaging into vesicles. (F) Electrical stimulation trains $(0.1 \mathrm{~Hz})$ were used to deplete axonal ATP. Repeated measurements were taken at $950 \mathrm{~nm}$ and presented as $\Delta \mathrm{F} / \mathrm{F}_{0}$; repetitive stimulation decreased axonal ATP. In the presence of the MAO-B inhibitior rasagiline (+MAO-Bi) ATP levels were further decreased and yet further with complex V inhibition (+oligomycin) (lines are median values, shading is interquartile range; control $n=8,+$ MAOBi $n=9$, and +oligomycin $n=6$ brain slices). (G) To better visualize the contribution of MAO-B to ATP generation, the measurements in the presence of rasagaline were subtracted from those in control aCSF to yield MAO-B dependent ATP (line represents the median MAO-B/aCSF differential, shading is interquartile range; $\mathrm{n}=9$ brain slices). (H) Pre-train: Inhibition of MAO-B or complex V (+oligomycin) both decreased the ATP:ADP bioenergetic index (Kruskal-Wallis test: aCSF vs rasagaline $\mathrm{p}=0.011$, aCSF vs oligomycin $\mathrm{p}=0.0005$; control $\mathrm{n}=7$; +MAOBi $\mathrm{n}=7$; +oligomycin $\mathrm{n}=6$ brain slices). Post-train: Electrical stimulation trains decreased ATP signal in all cases but the effect was more pronounced in the absence of mitochondrial ATP production (Kruskal-Wallis test: aCSF vs oligomycin $\mathrm{p}=0.0005$, control $\mathrm{n}=6$; +MAOBi $\mathrm{n}=6$, +oligomycin $\mathrm{n}=6$ ). Box-and-whisker plots depict median, quartiles, and range(I) Images of dopamine axons expressing dopamine biosensor dLight $1.3 \mathrm{~b}$ before and after electrical stimulation (1p, $350 \mu \mathrm{A}, 2 \mathrm{~ms})$; scale bar denotes $10 \mu \mathrm{M}$. (J) Release probability was interrogated using electrical stimulation to mimic tonic $(1 \mathrm{p}, 2 \mathrm{~ms}, 350 \mu \mathrm{A})$ or phasic dopamine release $(5 \mathrm{p} 100 \mathrm{~Hz}, 2 \mathrm{~ms}, 350 \mathrm{uA})$ in the presence of synaptic blockade (10 $\mu \mathrm{M}$ mecamylamine, $10 \mu \mathrm{M}$ sulpiride). Traces of quantified dLight fluorescence in response to tonic or phasic release stimulation. Line: median, shading $95 \% \mathrm{CI}$; control $\mathrm{n}=5$, + MAOBi $n=6$, and +oligomycin $n=4$ brain slices. (K) No statistically significant difference was seen in tonic firing in response to $+\mathrm{MAOBi}$ (rasagaline, $10 \mathrm{uM}$ ) or +oligomycin (10 $\mathrm{uM})$. Phasic firing was significantly decreased by $+\mathrm{MAOBi}$ and further decreased by +oligomycin (2-way ANOVA, 5p100Hz: control vs rasagaline $\mathrm{p}=0.015$, control vs oligomycin $\mathrm{p}<0.0001$, rasagaline vs oligomycin $\mathrm{p}=0.028$; control $\mathrm{n}=5,+\mathrm{MAOBi} \mathrm{n}=6$, and +oligomycin $\mathrm{n}=4$ brain slices). Stimulation increases release $(\mathrm{F}=169.63, \mathrm{DFd}=12$, $\mathrm{p}<0.0001)$. Drug affects release $(\mathrm{F}=10.07, \mathrm{DFd}=12, \mathrm{p}=0.003$. The drugs alter stimulus response $(\mathrm{F}=5.64, \mathrm{DFd}=12, \mathrm{p}=0.019)$. Box-and-whisker plots depict median, quartiles, and range. $* \mathrm{p}<0.05$. 